\title{
FUNDAMENTAL DOMAIN OF INVARIANT SETS AND APPLICATIONS
}

\author{
PENGFEI ZHANG
}

\begin{abstract}
Let $X$ be a compact metric space and $f: X \rightarrow X$ a homeomorphism on $X$. We construct a fundamental domain for the set with finite peaks for each cocycle induced by $\phi \in C(X, \mathbb{R})$. In particular we prove that if a partially hyperbolic diffeomorphism is accessible, then either the set with finite peaks for the Jacobian cocycle is of full volume, or the set of transitive points is of positive volume.
\end{abstract}

\section{INTRODUCTION}

In this paper we give a construction of fundament domains for some general subsets. More precisely let $X$ be a compact metric space, $f: X \rightarrow X$ a homeomorphism and $E$ be an $f$-invariant set. If there is an $f$-invariant Borel map $s: E \rightarrow E$ such that $s(x) \in \mathcal{O}(f, x)$, then the image of $s$ is called a fundamental domain of $E$. Take the North and South Poles Map $f: S^{2} \rightarrow S^{2}$ for example: the set $E=S^{2} \backslash\{N, S\}$ is $f$-invariant and $B(S, r) \backslash f B(S, r)$ is a fundamental domain of $E$ (for $r<1$ ).

In general let $\phi \in C(X, \mathbb{R})$ be a continuous function. This induces an additive cocycle $\left\{\phi_{n}: n \in \mathbb{Z}\right\}$ over $(X, f)$ which is given by

$$
\phi_{n}(x)= \begin{cases}\phi(x)+\cdots+\phi\left(f^{n-1} x\right), & n \geq 0 ; \\ -\phi\left(f^{n} x\right)-\cdots-\phi\left(f^{-1} x\right), & n<0 .\end{cases}
$$

Let $\Phi_{f}(x)=\sup _{n \in \mathbb{Z}} \phi_{n}(x)$ be the peak value at $x$. Then the cocycle $\left\{\phi_{n}\right\}$ is said to have finite peaks at a point $x \in X$, if $\left\{n \in \mathbb{Z}: \phi_{n}(x)=\Phi_{f}(x)\right\}$ is nonempty and finite. Denote by $H(f, \phi)$ the set of points with finite peaks.

Note that for some $\phi$ related to the dynamics, $H(f, \phi)$ can be quite large with respect to natural measures, see Section 4 and [6. Also $H(f, \phi)$ could be large in the sense of entropy, see Remark 1 and Section 5. We prove that there always exists a fundamental domain for this set:

Theorem A. Let $f: X \rightarrow X$ be a homeomorphism and $\phi \in C(X, \mathbb{R})$. Then there is an $f$-invariant Borel section $\pi: H(f, \phi) \rightarrow H(f, \phi)$. Equivalently, the image of $\pi$ is a fundamental domain of $H(f, \phi)$.

2000 Mathematics Subject Classification. Primary 37D30; Secondary 37D20, 37B40.

Key words and phrases. Fundamental domain, partial hyperbolicity, cocycle, transitivity, accessibility, essential saturation.

The work is partially supported by NNSF project (11071231). 
We also give some applications of our construction. Let $f: M \rightarrow M$ be a transitive diffeomorphism and $\operatorname{Tran}_{f}$ be the set of transitive points of $f$. It is well known that $\operatorname{Tran}_{f}$ is a residual subset (hence topologically large). But a residual subset could be measure-theoretically meagre (take the set of Liouville numbers for example).

There are some classical results about the measure-theoretical largeness of the transitive set. For example let $f: M \rightarrow M$ be a $C^{2}$ transitive Anosov diffeomorphism. Sinaĩ proved in [11] that there exists a unique Gibbs measure $\mu_{+}$with respect to $f$ whose basin $B\left(\mu_{+}, f\right)$ is of full volume: $m\left(B\left(\mu_{+}, f\right)\right)=1$. In particular $B\left(\mu_{+}, f\right) \subset \operatorname{Tran}_{f}$ since the support $\operatorname{supp}\left(\mu_{+}\right)=M$. So $m\left(\operatorname{Tran}_{f}\right)=1$ for every $C^{2}$ transitive Anosov diffeomorphism. See [2, 13] for recent results about the measure of transitive sets for general systems. We get similar estimates of $\operatorname{Tran}_{f}$ for accessible partially hyperbolic systems. Namely let $J_{f}(x)$ be the Jacobian of $f$ with respect to the Riemannian metric which induces $m$.

Theorem B. Let $f: M \rightarrow M$ be a $C^{2}$ partially hyperbolic diffeomorphism. If $f$ is essentially accessible and $m\left(H\left(f, \log J_{f}\right)\right)<1$, then $f$ is transitive and $m\left(\operatorname{Tran}_{f}\right) \geq 1-$ $m\left(H\left(f, \log J_{f}\right)\right)>0$.

Once again let $f$ be $C^{2}$ transitive Anosov diffeomorphism, $\mu_{+}$(respectively, $\mu_{-}$) be the unique Gibbs measure with respect to $f$ (respectively, $f^{-1}$ ). Denote the common measure by $\mu$ if $\mu_{+}=\mu_{-}$. Following dichotomy is proved by Gurevich and Oseledets [6]:

- either $\mu_{+} \neq \mu_{-}$: then $(m, f)$ is completely dissipative;

- or $\mu_{+}=\mu_{-}$: then $\mu$ is equivalent to $m$ and $(m, f)$ is ergodic.

We also get a partial generalization of Gurevich and Oseledets dichotomy to center bunched, essentially accessible partially hyperbolic systems.

Corollary C. Let $f: M \rightarrow M$ be a $C^{2}$ essentially accessible, center bunched partially hyperbolic diffeomorphism.

(1) Either $m\left(H\left(f, \log J_{f}\right)\right)=1$ : then $(m, f)$ is completely dissipative;

(2) or $m\left(H\left(f, \log J_{f}\right)\right)<1$ : then $m\left(H\left(f, \log J_{f}\right)\right)=0$ and $(m, f)$ is ergodic.

Finally we give a proof of the entropy largeness of the Birkhoff heteroclinic set $H_{f}(\mu, \nu)=$ $B(\mu, f) \cap B\left(\nu, f^{-1}\right)$ of two $f$-invariant measures $\mu, \nu$, which may be of interest in its own right. We are grateful to G. Liao for pointing out the asymmetry of Bowen dimensional entropy $h_{B}(f, \cdot)$.

Proposition D. Let $f: M \rightarrow M$ be a transitive Anosov diffeomorphism. Then for all $f$-invariant measures $\mu, \nu$, the entropy of $H_{f}(\mu, \nu)$ satisfies $h_{B}\left(f, H_{f}(\mu, \nu)\right)=h_{\mu}(f)$ and $h_{B}\left(f^{-1}, H_{f}(\mu, \nu)\right)=h_{\nu}(f)$.

In particular if $h_{\mu}(f) \neq h_{\nu}(f)$, then $h_{B}\left(f, H_{f}(\mu, \nu)\right) \neq h_{B}\left(f^{-1}, H_{f}(\mu, \nu)\right)$. 


\section{ACKNOWLEDGMENTS}

We thank Jon Aaronson, Shaobo Gan, Gang Liao, Lan Wen, Shuyun (Conan) Wu and Zhihong Xia for useful discussions. The author benefited a lot from Shaobo Gan and Gang Liao's suggestions. We thank Shuyun (Conan) Wu for sending me a copy of the paper [6].

\section{Fundamental DOMAIN OF SOME INVARIANT SUBSETS}

Let $X$ be a compact metric space, $f: X \rightarrow X$ a homeomorphism and $\phi \in C(X, \mathbb{R})$ be a continuous function. The induced cocycle $\left\{\phi_{n}: n \in \mathbb{Z}\right\}$ over $(X, f)$ is given by

$$
\phi_{n}(x)= \begin{cases}\phi(x)+\cdots+\phi\left(f^{n-1} x\right), & n \geq 0 ; \\ -\phi\left(f^{n} x\right)-\cdots-\phi\left(f^{-1} x\right), & n<0 .\end{cases}
$$

In particular $\phi_{0}(x) \equiv 0, \phi_{n+k}(x)=\phi_{n}(x)+\phi_{k}\left(f^{n} x\right)$ for all $n, k \in \mathbb{Z}$ and $x \in X$.

Definition 2.1. Let $f: X \rightarrow X$ be a homeomorphism, $\phi \in C(X, \mathbb{R})$ and $\Phi_{f}(x)=$ $\sup _{n \in \mathbb{Z}} \phi_{n}(x)$. The cocycle $\left\{\phi_{n}\right\}$ is said to have finite peaks at a point $x \in X$ if $\{n \in \mathbb{Z}$ : $\left.\phi_{n}(x)=\Phi_{f}(x)\right\}$ is nonempty and finite. Denote by $H(f, \phi)$ the set of points with finite peaks.

It is easy to see the set $H(f, \phi)$ is a Borel subset. And $H(f, \phi)=\emptyset$ if $\phi$ is constant. In the following we assume that $H(f, \phi) \neq \emptyset$.

Definition 2.2. The function $n_{f}$ of last peak time and the function $\pi$ of last peak position on $H(f, \phi)$ are defined as:

$$
n_{f}(x)=\sup \left\{n \in \mathbb{Z}: \phi_{n}(x)=\Phi_{f}(x)\right\}, \text { and } \pi(x)=f^{n_{f}(x)} x .
$$

Let $H_{N}=\left\{x \in X: \phi_{n}(x)<\Phi_{f}(x)\right.$ for all $n$ with $\left.|n|>N\right\}$ for each $N \geq 1$. It is clear that $H(f, \phi)=\bigcup_{N>1} H_{N}$ and $\Phi_{f}(x)=\max _{|n| \leq N} \phi_{n}(x)$ on $H_{N}$. So the set $H_{N}$ is a $G_{\delta^{-}}$subset and the function $\Phi_{f}$ is continuous on $H_{N}$. Also note that for each $x \in H_{N}$, $\phi_{n}(x)<\Phi_{f}(x)$ for all $|n|>N$ and hence $\left|n_{f}(x)\right| \leq N$. Moreover we have

Lemma 2.3. Let $f$ be a homeomorphism on $X, \phi \in C(X, \mathbb{R})$ and $H_{N}$ given as above. Then $n_{f}$ is upper semi-continuous on $H_{N}$ and the restriction $\left.\pi\right|_{H_{N}}$ is a Borel map.

Proof. (1). Let $x \in H_{N}$ and $x_{k} \in H_{N} \rightarrow x$. Note that $\left|n_{f}\left(x_{k}\right)\right| \leq N$ for all $k \geq 1$. Passing to a subsequence if necessary, we assume $n_{f}\left(x_{k}\right)=\hat{n}$ for all $k \geq 1$. Now we claim $n_{f}(x) \geq \hat{n}$ and hence $n_{f}$ is upper semi-continuous on $H_{N}$.

If this were not true, then $n_{f}(x)<\hat{n}$ and hence $\Phi_{f}(x)>\phi_{\hat{n}}(x)$. Since $\Phi_{f}$ is continuous on $H_{N}$, there exists $\delta>0$ such that $\Phi_{f}(y)>\phi_{\hat{n}}(y)$ for all $y \in H_{N} \cap B(x, \delta)$. In particular $\Phi_{f}\left(x_{k}\right)>\phi_{\hat{n}}\left(x_{k}\right)$ for all $k$ large, which contradict the assumption $\hat{n}=n_{f}\left(x_{k}\right)$. Therefore $\limsup \sup _{k \rightarrow \infty} n_{f}\left(x_{k}\right) \leq n_{f}(x)$. This finishes the proof of the claim and hence the first conclusion.

(2). Let $H(n)=\left\{x \in H_{N}: n_{f}(x)=n\right\}$. Clearly $H(n)$ is a Borel set and $\bigsqcup_{|n| \leq N} H(n)=$ $H_{N}$. Then $\left.\pi\right|_{H(n)}=\left.f^{n}\right|_{H(n)}$ is a Borel map for each $|n| \leq N$. So $\left.\pi\right|_{H_{N}}$ is also Borel. 
Theorem 2.4. Let $f: X \rightarrow X$ be a homeomorphism and $\phi \in C(X, \mathbb{R})$. Then the map $\pi$ is an $f$-invariant Borel section on $H(f, \phi)$. Equivalently, the image of $\pi$ is a fundamental domain of $H(f, \phi)$.

Proof. Let $k \in \mathbb{Z}$. Since $\phi_{n+k}=\phi_{k}+\phi_{n} \circ f^{k}$ for all $n \in \mathbb{Z}$, we see that

$$
\Phi_{f}(x)=\sup _{n \in \mathbb{Z}} \phi_{n+k}(x)=\sup _{n \in \mathbb{Z}} \phi_{n}\left(f^{k} x\right)+\phi_{k}(x)=\Phi_{f}\left(f^{k} x\right)+\phi_{k}(x) .
$$

For (2.2) we see that for every $x \in H(f, \phi)$ :

- $\phi_{n+k}(x)=\Phi_{f}(x)$ if and only if $\phi_{n}\left(f^{k} x\right)=\Phi_{f}\left(f^{k} x\right)$;

- moreover $n_{f}(x)=n_{f}\left(f^{k} x\right)+k$.

Then we have

$$
\pi\left(f^{k} x\right)=f^{n_{f}\left(f^{k} x\right)}\left(f^{k} x\right)=f^{n_{f}\left(f^{k} x\right)+k}(x)=f^{n_{f}(x)}(x)=\pi(x) .
$$

Thus $\pi$ is $f$-invariant and $\pi(x) \in \mathcal{O}_{f}(x)$. By Lemma 2.3 we see that $\pi: H(f, \phi) \rightarrow H(f, \phi)$ is an $f$-invariant Borel section and its image $W=\pi(H(f, \phi))$ is a fundamental domain of $H(f, \phi)$. This completes the proof.

\section{Applications: topological Systems}

Let $f: X \rightarrow X$ be a homeomorphism and $\mathcal{M}(f)$ the $f$-invariant probability measures. Let $\nu_{x, n}=\frac{1}{n} \sum_{k=0}^{n-1} \delta_{f^{k} x}$ be the Birkhoff average along the orbit segment $\left\{x, \cdots, f^{n-1} x\right\}$. Then the basin $B(\mu, f)$ of $\mu$ with respect to $f$ is defined as $B(\mu, f)=\left\{x \in X: \nu_{x, n} \rightarrow\right.$ $\mu$ as $n \rightarrow+\infty\}$, which can be viewed as the Birkhoff stable set of the measure $\mu$. In this spirit we give the following:

Definition 3.1. Let $\mu, \nu \in \mathcal{M}(f)$ be two distinct invariant measures. The set of the Birkhoff heteroclinid] points of the pair $(\mu, \nu)$, denoted by $H_{f}(\mu, \nu)$, is defined as $H_{f}(\mu, \nu)=$ $B(\mu, f) \cap B\left(\nu, f^{-1}\right)$.

Remark 1. Let $f: M \rightarrow M$ be a transitive Anosov diffeomorphism. By Limit Shadowing Property we see that $B(\mu, f) \neq \emptyset$ for all invariant measure $\mu$, so is $B\left(\nu, f^{-1}\right)$. Also note that $B(\mu, f)$ is saturated by stable manifolds and $B\left(\nu, f^{-1}\right)$ is saturated by unstable manifolds. Therefore $H_{f}(\mu, \nu)$ is dense for all invariant measures $(\mu, \nu)$. In fact $h_{B}\left(f, H_{f}(\mu, \nu)\right)=h_{\mu}(f)$ where $h_{B}(f, E)$ be Bowen's dimensional entropy on noncompact sets (see Section 5). So there are many invariant pairs with large heteroclinic sets $H_{f}(\mu, \nu)$.

The following theorem provides a fundamental domain of the Birkhoff heteroclinic set:

Theorem 3.2. Let $(X, f)$ be given as above, and $\mu, \nu \in \mathcal{M}(f)$ with $H_{f}(\mu, \nu) \neq \emptyset$. Then there exists an $f$-invariant, Borel section $s$ on $H_{f}(\mu, \nu)$ and its image is a fundamental domain of $H_{f}(\mu, \nu)$.

\footnotetext{
${ }^{1}$ If we denote $B^{s}(\mu, f)=B(\mu, f)$ and $B^{u}(\nu, f)=B\left(\nu, f^{-1}\right)$, then $H_{f}(\mu, \nu)=B^{s}(\mu, f) \cap B^{u}(\nu, f)$.
} 
Proof. Since $\mu \neq \nu$, there exists a continuous function $\phi: X \rightarrow \mathbb{R}$ such that $\int_{X} \phi d \mu \neq$ $\int_{X} \phi d \nu$. Replacing $\phi$ by $a \phi+b$ if necessary, we assume that

$$
\int_{X} \phi d \mu=-1<0<1=\int_{X} \phi d \nu
$$

For each point $x \in H_{f}(\mu, \nu)=B(\mu, f) \cap B\left(\nu, f^{-1}\right)$ we see

$$
\begin{aligned}
\lim _{n \rightarrow+\infty} \frac{1}{n} \phi_{n}(x) & =\lim _{n \rightarrow+\infty} \frac{1}{n} \sum_{k=0}^{n-1} \phi\left(f^{k} x\right)=\int_{X} \phi d \mu=-1, \\
\lim _{n \rightarrow+\infty} \frac{1}{n} \phi_{-n}(x) & =\lim _{n \rightarrow+\infty} \frac{1}{n} \sum_{k=1}^{n}-\phi\left(f^{-k} x\right)=-\int_{X} \phi d \nu=-1 .
\end{aligned}
$$

Therefore $\phi_{n}(x)<0=\phi_{0}(x)$ for all $n$ with $|n|$ large. So $x \in H(f, \phi)$. Thus $H_{f}(\mu, \nu) \subset$ $H(f, \phi)$ and the restriction of $\pi$ (given by (2.1) ) to $H_{f}(\mu, \nu)$ provides the $f$-invariant section by Theorem 2.4. This completes the proof.

Similarly we can define the Birkhoff homoclinic set $H_{f}(\mu)=B(\mu, f) \cap B\left(\mu, f^{-1}\right)$. We first note that there is an obstruction for the existence of fundamental domain of Birkhoff homoclinic sets for ergodic measures:

Proof. Let $\mu$ be an ergodic measure. Then $\mu(B(\mu, f))=\mu\left(B\left(\mu, f^{-1}\right)\right)=1$ by Birkhoff ergodic theorem. So $\mu\left(H_{f}(\mu)\right)=1$. If there were some fundamental domain $W$ of $H_{f}(\mu)$, then either $\mu(W)=0$ (forces $\mu\left(H_{f}(\mu)\right)=0$ ) or $\mu(W)>0$ (forces $\mu\left(H_{f}(\mu)\right)=\infty$ ), contradicts $\mu\left(H_{f}(\mu)\right)=1$. So there do not exist any fundamental domain of $H_{f}(\mu)$.

Note that $\mu(B(\mu, f))=0$ for general $\mu \in \mathcal{M}(f) \backslash \mathcal{M}^{e}(f)$. So the obstruction no longer exists if $\mu$ is not ergodic. Moreover the basin $H_{f}(\mu)$ could be large in the sense of entropy. In fact $h_{B}\left(f, H_{f}(\mu)\right)=h_{\mu}(f)$ if $f$ is a transitive Anosov diffeomorphism (by Remark 1). We don't know if one can find a fundamental domain of $H_{f}(\mu)$ for these measures.

Now we give a simple corollary which will be used in next section. Let $X$ be a compact metric space and $\mu$ be a probability measure on $X$. Let $f: X \rightarrow X$ be a homeomorphism (may not preserves $\mu$ ).

Definition 3.3. Let $W$ be a measurable subset of positive $\mu$-measure. Then $E$ is said to be wandering with respect to $(\mu, f)$ if $f^{n} W, n \in \mathbb{Z}$ are mutually disjoint. The dissipative part $D_{f}$ of the system $(X, \mu, f)$ is the measurable union of the collection of measurable wandering sets with respect to $(\mu, f)$. The set $C_{f}=X \backslash D_{f}$ is called the conservative part of $(\mu, f)$. The partition $\left\{C_{f}, D_{f}\right\}$ is called the Hopf decomposition of $(\mu, f)$.

Note that $D_{f}=\emptyset$ if every measurable set of positive measure is not wandering. See [7, 8, 1, 9] for more details.

Assume there exists a continuous function $\phi: X \rightarrow \mathbb{R}$ such that $\mu(f E)=\int_{E} e^{\phi(x)} d \mu(x)$ for each measurable subset $E \subset M$. The function $e^{\phi}$ is called the Jacobian of $f$ with 
respect to $\mu$. As a byproduct of Theorem 2.4, we give a proof of a very special case of [9, Corolary 24] (simple and regular action) without using Rokhlin disintegration theorem:

Corollary 3.4. Let $f: X \rightarrow X$ be a homeomorphism and $\mu$ be a Borel measure with Jacobian $J_{f}=e^{\phi}$, where $\phi$ is a continuous function on $X$. Then the dissipative part $D_{f}$ of $(\mu, f)$ satisfies $\mu\left(D_{f} \Delta H(f, \phi)\right)=0$.

Proof. (1). To show $\mu\left(D_{f} \backslash H(f, \phi)\right)=0$, it suffices to show that for each wandering set $W, \sum_{n \in \mathbb{Z}} e^{\phi_{n}(x)}<+\infty$ for $\mu$-a.e. $x \in W$. This is true since

$$
\int_{W} \sum_{n \in \mathbb{Z}} e^{\phi_{n}(x)} d m(x)=\sum_{n \in \mathbb{Z}} \int_{f^{n} W} d m(x)=m\left(\bigcup_{n \in \mathbb{Z}} f^{n} W\right) \leq 1 .
$$

(2). Now we show $\mu\left(H(f, \phi) \backslash D_{f}\right)=0$. It is trivial if $\mu(H(f, \phi))=0$. Then assume $\mu(H(f, \phi))>0$. Let $W$ be the fundamental domain of $H(f, \phi)$ given by Theorem 2.4 . Then $\mu(W)>0$ and $W$ is wandering. So $H(f, \phi) \subset D_{f}$.

\section{Applications: Smooth Systems}

In this section we give some estimates about the transitive sets of partially hyperbolic systems. Let $M$ be a compact Riemannian manifold without boundary. Recall that $x \in M$ is a transitive point of $f$ if its orbit $\mathcal{O}_{f}(x)$ is dense on $M$. Denote by $\operatorname{Tran}_{f}$ be the set of transitive points.

A $C^{r}$ diffeomorphism $f: M \rightarrow M$ is said to be partially hyperbolic if there are a $T f$ invariant splitting of $T M=E^{s} \oplus E^{c} \oplus E^{u}$, a smooth Riemannian metric $g$ on $M$ and positive constants $\nu, \tilde{\nu}, \gamma$ and $\tilde{\gamma}$ with $\nu, \tilde{\nu}<1$ and $\nu<\gamma \leq \tilde{\gamma}^{-1}<\tilde{\nu}^{-1}$ such that, for all $x \in M$ and for all unit vectors $v \in E_{x}^{s}, w \in E_{x}^{c}$ and $v^{\prime} \in E_{x}^{u}$,

$$
\|T f(v)\| \leq \nu<\gamma \leq\|T f(w)\| \leq \tilde{\gamma}^{-1}<\tilde{\nu}^{-1} \leq\left\|T f\left(v^{\prime}\right)\right\|
$$

We assume that both $E^{s}$ and $E^{u}$ are nontrivial and continuous. It is well known that $E^{s}$ and $E^{u}$ are uniquely integrable and tangent to the stable foliation $\mathcal{W}^{s}$ and the unstable foliation $\mathcal{W}^{u}$ respectively 2 . Let $m$ be the normalized measure induced by the Riemannian metric $g$ on $M$.

Definition 4.1. Let $A$ be a measurable subset of $M$. Then $A$ is said to be $s$-saturated if for each $x \in A, W^{s}(x) \subset A$. Similarly we can define $u$-saturated sets. Then the set $A$ is bi-saturated if it is $s$-saturated and $u$-saturated.

The following is slightly general version of above one:

Definition 4.2. Let $A$ be a measurable subset of $M$. Then $A$ is said to be essentially $s$-saturated if there exists an $s$-saturated set $A^{s}$ with $m\left(A \Delta A^{s}\right)=0$. Similarly we can define essentially $u$-saturated sets. The set $A$ is essentially bi-saturated if there exists a bi-saturated set $A^{s u}$ with $m\left(A \Delta A^{s u}\right)=0$, and bi-essentially saturated if $A$ is essentially $s$-saturated and essentially $u$-saturated.

\footnotetext{
${ }^{2}$ Although these foliations may not be smooth, they are transversal absolutely continuous with $C^{r}$ leaves.
} 
It is worth to point out that there is a subtle difference between essential bi-saturation and bi-essential saturation, see [4].

Definition 4.3. A partially hyperbolic diffeomorphism $f: M \rightarrow M$ is said to be accessible if each nonempty bi-saturated set is the whole manifold $M$. The map $f$ is essentially accessible if every measurable bi-saturated set has either full or zero volume.

Dolgopyat and Wilkinson proved in [5] that accessibility holds on a $C^{1}$-open and $C^{1}$ dense subset of partially hyperbolic systems.

Now we are ready to prove the following proposition:

Proposition 4.4. Let $f: M \rightarrow M$ be a $C^{2}$ partially hyperbolic diffeomorphism and $C_{f}$ the conservative part of $(m, f)$. Assume $m\left(C_{f}\right)>0$.

(1) Then every $f$-invariant subset $E \subset C_{f}$ is bi-essentially saturated.

(2) Moreover if $f$ is essentially accessible, then m-a.e. $x \in C_{f}$ is a transitive point. In particular $m\left(\operatorname{Tran}_{f}\right) \geq m\left(C_{f}\right)>0$.

Note that $m\left(C_{f}\right)+m\left(H\left(f, \log J_{f}\right)\right)=m\left(C_{f}\right)+m\left(D_{f}\right)=1$ by Corollary 3.4 . So Theorem $\mathrm{B}$ follows from this proposition. We need the following :

Halmos Recurrence Theorem (Theorem 1.1.1 in [1]). Let $C_{f}$ be the conservative part of $(m, f)$. Then for every measurable subset $A \subset C_{f}, \sum_{n>1} 1_{A}\left(f^{n} x\right)=+\infty$ for $m$-a.e. $x \in A$. In other words, $m$-a.e. $x \in A$ will return to $A$ infinitely many times.

Proof of the first conclusion. Let $E \subset C_{f}$ be an $f$-invariant subset. We first show that $E$ is essentially $s$-saturated. Note that for each $x \in M$, the stable manifold $W^{s}(x)$ is a $C^{2}$ immersed submanifold. Denote by $m_{W^{s}(x)}$ (by $m_{s}$ for short) the leaf volume induced by the restricted Riemannian metric on $W^{s}(x)$.

Note that the proof of Lemma 4.1 in [12] also works for our case. The only difference is that we use Halmos Recurrence Theorem, instead of Poincaré Recurrence Theorem. So there exists a measurable subset $A \subset E$ with $m(E \backslash A)=0$ such that

$$
m_{s}\left(W^{s}(x) \backslash E\right)=0 \text {, for each } x \in C \text {. }
$$

To construct an $s$-saturate of $E$, we need the following fact, which follows from the continuity of the foliation $\mathcal{W}^{s}$ :

- If $K$ is closed subset of $M$, then $\bigcup_{x \in K} \overline{W_{R}^{s}(x)}$ is closed for all $R>0$.

Now let $K_{n} \subset K_{n+1} \subset \cdots \subset C$ be an increasing sequence of compact subsets with $m\left(C \backslash K_{n}\right) \rightarrow 0$. It is easy to see that the set $C^{s}=\bigcup_{n \geq 1} \bigcup_{x \in K_{n}} \overline{W_{n}^{s}(x)}$ is measurable, $s$-saturated and

a) $m\left(E \backslash C^{s}\right)=0$ since $m\left(E \backslash C^{s}\right) \leq m\left(E \backslash K_{n}\right)=m\left(C \backslash K_{n}\right) \rightarrow 0$ as $n \rightarrow \infty$.

b) $m\left(C^{s} \backslash E\right)=0$ by (4.2) and by absolute continuity of $\mathcal{W}^{s}$.

So $m\left(E \Delta C^{s}\right)=0$ for an $s$-saturated set $C^{s}$. Therefore $E$ is essentially $s$-saturated. The essential $u$-saturate property of $E$ follows similarly. This finishes the proof. 
To prove the second conclusion, we first show that for each open ball $B, \mathcal{O}(x) \cap B \neq \emptyset$ for $m$-a.e. point $x \in C_{f}$. To the end we consider $G(B)$, the subset of points $x$ which has a neighborhood $U$ of $x$ such that $\mathcal{O}(y) \cap B \neq \emptyset$ for $m$-a.e. $y \in U \cap C_{f}$. Evidently $G(B)$ is a nonempty open subset (and $f$-invariant). Note that we can replace $C_{f}$ by its $s$-saturate $C^{s}$ in the definition of $G(B)$ since $m\left(C_{f} \Delta C^{s}\right)=0$.

Lemma 4.5. The set $G(B)$ is bi-saturated and $m(G(B))=1$.

Proof. Let us prove $G(B)$ is $s$-saturated. It suffices to show that $q \in G(B)$ for each $q \in W_{\delta}^{s}(p)$ and each $p \in G(B)$, where the size $\delta$ is fixed. So the justification lies in a local foliation box $Z$ of $\mathcal{W}^{s}$ around $p$.

For a point $x \in Z$, denote $W_{Z}^{s}(x)$ the component of $W^{s}(x) \cap Z$ that contains $x$. Since $p \in G(B)$, there exists a small neighborhood $U$ of $p$ with $\mathcal{O}(y) \cap B \neq \emptyset$ for $m$-a.e. $y \in U \cap C^{s}$.

Let $R$ be the set of recurrent points in $U \cap C^{s}$ whose orbits enter $B$. Clearly $m(U \cap$ $\left.C^{s} \backslash R\right) \leq m\left(C_{f} \backslash R\right)=0$. So we can pick a smooth transverse $\tau \subset U$ of $\mathcal{W}_{Z}^{s}$ such that $\tau \cap W_{U}^{s}(p) \neq \emptyset$ and $m_{\tau}\left(C^{s} \backslash R\right)=0$, where $m_{\tau}$ is the induced volume on $\tau$ (note that $C^{s}$ is not only essentially $s$-saturated, but $s$-saturated). Now we have the set $\bigcup_{x \in \tau \cap R} W_{Z}^{s}(x)$ has full $m$-measure in the set $\bigcup_{x \in \tau \cap C^{s}} W_{Z}^{s}(x)=\left(\bigcup_{x \in \tau} W_{Z}^{s}(x)\right) \cap C^{s}$.

The set $\bigcup_{x \in \tau} W_{Z}^{s}(x)$ contains an open neighborhood $V$ of $q$. Moreover $\mathcal{O}(y) \cap B \neq \emptyset$ for $m$-a.e. $y \in V \cap C^{s}$ and therefore $q \in G(B)$. This implies $G(B)$ is $s$-saturated. Similarly $G(B)$ is $u$-saturated and hence $m(G(B))=1$ by the essential accessibility of $f$.

The rest of the proof follows closely from the proof of Theorem 5.5 in [13] and hence is omitted here. So $m$-a.e. $x \in C_{f}$ is a transitive point and $m\left(\operatorname{Tran}_{f}\right) \geq m\left(C_{f}\right)>0$. This completes the proof of Proposition 4.4 .

To get sharper results we need the following definition:

Definition 4.6. A partially hyperbolic diffeomorphism $f$ is center bunched if the constants $\nu, \tilde{\nu}$ and $\gamma, \tilde{\gamma}$ given in (4.1) can be chosen so that: $\nu<\gamma \tilde{\gamma}$ and $\tilde{\nu}<\gamma \tilde{\gamma}$.

Proposition 4.7 (Corollary 5.2 in [4]). Let $f: M \rightarrow M$ be a $C^{2}$ center bunched partially hyperbolic diffeomorphism. Then every measurable bi-essentially saturated subset is essentially bi-saturated.

The following is a direct corollary of Proposition 4.4 and [4, Corollary 5.2], which provides a partial generalization of Gurevich and Oseledets dichotomy:

Corollary 4.8. Let $f: M \rightarrow M$ be a $C^{2}$ essentially accessible, center bunched partially hyperbolic diffeomorphism.

(1) Either $D_{f}=M$ : then $(m, f)$ is completely dissipative,

(2) or $C_{f}=M$ : then $(m, f)$ is ergodic.

Recall that $(m, f)$ is said to be ergodic if every measurable, $f$-invariant subset $E$ satisfies $m(E)=0$ or 1 . Note that $m$ may not be $f$-invariant. 
Proof. Assume $m\left(C_{f}\right)>0$. Then $C_{f}$ is bi-essentially saturated and hence also essentially bi-saturated by Proposition 4.7. So $m\left(C_{f}\right)=1$ by the essential accessibility of $f$. Hence $D_{f}=\emptyset$ and $C_{f}=M$.

Now let $E \subset M$ be an $f$-invariant subset with $m(E)>0$. Since $C_{f}=M$, we get that $E$ is also bi-essentially saturated by Proposition 4.4. So $E$ is essentially bi-saturated by Proposition 4.7 and $m(E)=1$ by the essential accessibility of $f$. This shows that $(m, f)$ is ergodic.

\section{LARGeness of HeterocliniC Sets}

In this section we prove that the heteroclinic sets can have large entropy. We first give the definition of the Bowen dimensional entropy $h_{B}(f, \cdot)$ for noncompact subsets [3] with respect to a homeomorphism $f: X \rightarrow X$. For $k \geq 1$ and $x, y \in X$, let $d_{k}(x, y)=$ $\max \left\{d\left(f^{i} x, f^{i} y\right): 0 \leq i<k\right\}$, and let $B(x, r, k)=\left\{y \in X: d_{k}(x, y)<r\right\}$ be the Bowen ball of radius $r>0$.

Let $E \subseteq X$ and $t \geq 0$. For any $\epsilon>0$ and $n \geq 1$, denote

$$
M_{n}(f, E, t, \epsilon)=\inf \left\{\sum_{i \geq 1} e^{-t n_{i}}: \bigcup_{i \geq 1} B\left(x_{i}, r, n_{i}\right) \supseteq E \text { and } n_{i} \geq n \text { for each } i \geq 1\right\} .
$$

Since $M_{n}(f, E, t, \epsilon)$ is increasing with respect to $n \in \mathbb{N}$, the limit

$$
M(f, E, t, \epsilon)=: \lim _{n \rightarrow \infty} M_{n}(f, E, t, \epsilon)
$$

is well defined. It is clear that $M(f, E, t, \epsilon) \leq M(f, E, s, \epsilon)$ if $t \geq s \geq 0$ and $M(f, E, t, \epsilon) \notin$ $\{0,+\infty\}$ for at most one point $t \geq 0$. Then define

$$
h_{B}(T, E, \epsilon)=\inf \{t \geq 0: M(f, E, t, \epsilon)=0\}=\sup \{t \geq 0: M(f, E, t, \epsilon)=+\infty\} .
$$

The Bowen dimensional entropy of $E$ is $h_{B}(f, E)=\lim _{\epsilon \rightarrow 0} h_{B}(T, E, \epsilon)$. Note that

$$
h_{B}\left(f, \bigcup_{i \geq 1} E_{i}, \epsilon\right)=\max _{i \geq 1} h_{B}\left(f, E_{i}, \epsilon\right) \text {, and } h_{B}\left(f, \bigcup_{i \geq 1} E_{i}\right)=\max _{i \geq 1} h_{B}\left(f, E_{i}\right) \text {. }
$$

Let $f: M \rightarrow M$ be a transitive Anosov diffeomorphism and $P=\left[W_{l o c}^{s}(x), W_{l o c}^{u}(x)\right]$ be a small rectangle. Let $y, z \in W_{l o c}^{s}(x)$ and $h_{y, z}^{s}: W_{P}^{u}(y) \rightarrow W_{P}^{u}(z)$ be the local stable holonomy (homeomorphism) with respect to $W^{s}$.

Lemma 5.1. Let $E \subset W_{P}^{u}(x)$. Then $h_{B}\left(f, h_{x, y}^{s} E, 2 \epsilon\right) \leq h_{B}(f, E, \epsilon) \leq h_{B}\left(f, h_{x, y}^{s} E, \epsilon / 2\right)$ for all $\epsilon>0$ and for all $y \in W_{l o c}^{s}(x)$. In particular $h_{B}\left(f, h_{x, y}^{s} E\right)=h_{B}(f, E)$ for all $y \in W_{l o c}^{s}(x)$.

Proof. Step 1. Let $\epsilon>0$. Note that $f B(z, \epsilon, n+1) \subset B(f z, \epsilon, n)$ always holds. So $M_{n}(f, f E, t, \epsilon) \leq e \cdot M_{n+1}(f, E, t, \epsilon)$ and hence $h_{B}(f, f E, \epsilon) \leq h_{B}(f, E, \epsilon)$. Now we show the other direction. 
Let $n \geq 1$ and $\left\{B\left(z_{l}, \epsilon, n_{l}\right): n_{l} \geq n\right\}$ be a covering of $f E$. Then $B\left(f^{-1} z_{l}, \epsilon\right) \cap E \supset$ $f^{-1}\left(B\left(z_{l}, \epsilon\right) \cap f E\right)$ (since $\left.f^{-1}\right|_{f W_{P}^{u}(x)}$ is contracting) and hence

$$
\begin{aligned}
B\left(f^{-1} z_{l}, \epsilon, n_{l}+1\right) \cap E & =B\left(f^{-1} z_{l}, \epsilon\right) \cap f^{-1} B\left(z_{l}, \epsilon, n_{l}\right) \cap E \\
& \supset f^{-1}\left(B\left(z_{l}, \epsilon\right) \cap f E\right) \cap f^{-1} B\left(z_{l}, \epsilon, n_{l}\right) \supset f^{-1}\left(B\left(z_{l}, \epsilon, n_{l}\right) \cap f E\right) .
\end{aligned}
$$

So $\left\{B\left(f^{-1} z_{l}, \epsilon, n_{l}+1\right): n_{l} \geq n\right\}$ be a covering of $E$. This implies that $M_{n}(f, f E, t, \epsilon) \geq$ $e \cdot M_{n+1}(f, E, t, \epsilon)$ and $h_{B}(f, f E, \epsilon) \geq h_{B}(f, E, \epsilon)$. So $h_{B}(f, f E, \epsilon)=h_{B}(f, E, \epsilon)$ for all $\epsilon>0$. Breaking $f^{k} E$ into small pieces, applying (5.2) and using inductive argument, we see $h_{B}\left(f, f^{k} E, \epsilon\right)=h_{B}(f, E, \epsilon)$ all $k \geq 1$.

Step 2. Pick $\delta>0$ such that $d\left(h_{x, y}^{s}(p), p\right)<\delta$ for all $p \in W_{P}^{u}(x)$. Iterating forward by $f^{k}$, we get a new homeomorphism $h_{k}^{s}: f^{k} W_{P}^{u}(x) \rightarrow f^{k} W_{P}^{u}(y)$ induced by $f^{k} \circ h_{x, y}^{s} \circ f^{-k}$. Note that $d\left(h_{k}^{s}\left(f^{k} p\right), f^{k} p\right)<\lambda^{k} \delta$ for all $p \in W_{P}^{u}(x)$, where $\lambda \in(0,1)$ is the contraction rate on $W^{s}$. Also note that $f^{k} \circ h_{x, y}^{s} E=h_{k}^{s} \circ f^{k} E$.

Step 3. Let $E \subset W_{P}^{u}(x), \epsilon>0$ and $t>h=h_{B}(f, E, \epsilon)$. By Step 1 we see $h_{B}\left(f, f^{k} E, \epsilon\right)<t$ for all $k \geq 1$.

Pick $N=N_{\epsilon, k} \geq 1$ large such that for each $n \geq N$, there exists a countable cover of $f^{k} E$, say $\left\{B\left(z_{l}, \epsilon, n_{l}\right): z_{l} \in f^{k} E, n_{l} \geq n\right\}$, such that $\sum_{l \geq 1} e^{-t n_{l}}<M_{n}\left(f, f^{k} E, t, \epsilon\right)+1$.

For each $q \in f^{k} h_{x, y}^{s} E$, we know $q=h_{k}^{s}(p) \in h_{k}^{s} f^{k} E$ where $p \in f^{k} E \cap B\left(z_{l}, \epsilon, n_{l}\right)$ for some $l \geq 1$. So

$$
d_{n}\left(h_{k}^{s} z_{l}, q\right) \leq d_{n}\left(h_{k}^{s} z_{l}, z_{l}\right)+d_{n}\left(z_{l}, p\right)+d_{n}(p, q) \leq 2 \lambda^{k} \delta+\epsilon .
$$

Therefore the collection $\left\{B\left(h_{k}^{s} z_{l}, 2 \lambda^{k} \delta+\epsilon, n_{l}\right): n_{l} \geq n\right\}$ forms a cover $f^{k} h_{x, y}^{s} E$ and

$$
M_{n}\left(f, f^{k} h_{x, y}^{s} E, t, 2 \lambda^{k} \delta+\epsilon\right) \leq \sum_{l \geq 1} e^{-t n_{l}}<M_{n}\left(f, f^{k} E, t, \epsilon\right)+1 .
$$

Passing $n$ to infinity, we see $M\left(f, f^{k} h_{x, y}^{s} E, t, 2 \lambda^{k} \delta+\epsilon\right) \leq 1$. Hence for every every $k \geq 1$,

- $h_{B}\left(f, h_{x, y}^{s} E, 2 \lambda^{k} \delta+\epsilon\right)=h_{B}\left(f, f^{k} h_{x, y}^{s} E, 2 \lambda^{k} \delta+\epsilon\right) \leq t$.

Picking $k$ large with $2 \lambda^{k} \delta<\epsilon$, we see $h_{B}\left(f, h_{x, y}^{s} E, 2 \epsilon\right) \leq t$. Then passing $t$ to $h$, we see $h_{B}\left(f, h_{x, y}^{s} E, 2 \epsilon\right) \leq h=h_{B}(f, E, \epsilon)$. Note that $h_{y, x}^{s} \circ h_{x, y}^{s}=I d$. So we can prove $h_{B}(f, E, \epsilon) \leq h_{B}\left(f, h_{x, y}^{s} E, \epsilon / 2\right)$ for all $\epsilon>0$. Finally passing $\epsilon$ to zero and applying Squeeze Theorem, we see $h_{B}\left(f, h_{x, y}^{s} E\right)=h_{B}(f, E)$ for every $y \in W_{l o c}^{s}(x)$. This completes the proof.

Lemma 5.2. Let $P=\left[W_{\text {loc }}^{s}(x), W_{\text {loc }}^{u}(x)\right]$ be a rectangle and $E \subset P$ be $W_{P}^{s}$-saturated, then

$$
h_{B}\left(f, E \cap W_{P}^{u}(y), \epsilon / 2\right) \leq h_{B}(f, E, \epsilon) \leq h_{B}\left(f, E \cap W_{P}^{u}(y), \epsilon / 2\right)
$$

for every $\epsilon>0$ and for every $y \in W_{l o c}^{s}(x)$. In particular $h_{B}(f, E)=h_{B}\left(f, E \cap W_{P}^{u}(y)\right)$ for every $y \in W_{l o c}^{s}(x)$

Proof. Let $\epsilon>0$ and $y \in W_{l o c}^{s}(x)$ be given. Clearly $E \cap W_{P}^{u}(z)=h_{y, z}^{s}\left(E \cap W_{P}^{u}(z)\right)$. Then by Lemma 5.1 we see $h_{B}\left(f, E \cap W_{P}^{u}(z), \epsilon\right) \leq h=h_{B}\left(f, E \cap W_{P}^{u}(y), \epsilon / 2\right)$ for every 
$z \in W_{\text {loc }}^{s}(x)$. Let $t>h$ be fixed. Pick a $\delta$-dense subset $\left\{x_{1}, \cdots, x_{d}\right\} \subset W_{P}^{s}(x)$. Then $h_{B}\left(f, E \cap W_{P}^{u}\left(x_{j}\right), \epsilon\right)<t$ for all $j=1, \cdots, d$.

There exists $N=N_{\epsilon, d} \geq 1$ such that for each $n \geq N$, there exists a countable cover of $E \cap W_{P}^{u}\left(x_{j}\right)$, say $\mathcal{C}_{j, n}=\left\{B\left(z_{l}^{j}, \epsilon, n_{l}^{j}\right): z_{l}^{j} \in E \cap W_{P}^{u}\left(x_{j}\right), n_{l}^{j} \geq n\right\}$, such that

$$
\sum_{l \geq 1} e^{-t n_{l}^{j}}<M_{n}\left(f, E \cap W_{P}^{u}\left(x_{j}\right), t, \epsilon\right)+\frac{1}{2 d}<\frac{1}{d} .
$$

For each $y \in W_{\epsilon}^{s}\left(x_{j}\right)$, denote the stable holonomy by $h_{j, y}^{s}: W_{P}^{u}\left(x_{j}\right) \rightarrow W_{P}^{u}(y)$ and $s(\delta)=$ $d\left(h_{j, y}^{s}, I d\right)$. For each $q \in E \cap W_{P}^{u}(y)$ there exists $p \in E \cap W_{P}^{u}\left(x_{j}\right)$ with $h_{j, y}^{s}(p)=q$. Also $p \in B\left(z_{l}^{j}, \epsilon, n_{l}\right)$ for some $l \geq 1$. So

$$
d_{n}\left(z_{l}^{j}, q\right) \leq d_{n}\left(z_{l}^{j}, p\right)+d_{n}(p, q) \leq s(\delta)+\epsilon .
$$

So $\left\{B\left(z_{l}^{j}, s(\delta)+\epsilon, n_{l}^{j}\right): l \geq 1\right\}$ covers $E \cap W_{P}^{u}\left(W_{\epsilon}^{s}\left(x_{j}\right)\right)$ for each $j=1, \cdots, d$. Therefore $\left\{B\left(z_{l}^{j}, s(\delta)+\epsilon, n_{l}^{j}\right): l \geq 1,1 \leq j \leq d\right\}$ covers $E$ and

$$
M_{n}(f, E, t, s(\delta)+\epsilon) \leq \sum_{j=1}^{d} \sum_{l \geq 1} e^{-t n_{l}^{j}} \leq \sum_{j=1}^{d} 1 / d=1 .
$$

Passing $n$ to infinity, we see $M(f, E, t, s(\delta)+\epsilon) \leq 1$ and hence $h_{B}(f, E, s(\delta)+\epsilon) \leq t$ for every $\delta>0$.

Passing $\delta$ to zero, we see $s(\delta)<\epsilon$ and $h_{B}(f, E, 2 \epsilon) \leq t$. Then passing $t$ to $h$, we see $h_{B}(f, E, 2 \epsilon) \leq h=h_{B}\left(f, E \cap W_{P}^{u}(y), \epsilon / 2\right)$. Clearly $h_{B}(f, E, 2 \epsilon) \geq h_{B}\left(f, E \cap W_{P}^{u}(y), 2 \epsilon\right)$. Finally passing $\epsilon$ to zero and applying Squeeze Theorem we get $h_{B}(f, E)=h_{B}(f, E \cap$ $\left.W_{P}^{u}(y)\right)$ for every $y \in W_{l o c}^{s}(x)$. This finishes the proof.

Now we give the proof of Proposition D.

Proof of Proposition D. Let $f: M \rightarrow M$ be a transitive Anosov diffeomorphism. Note that $f$ satisfies Specification Property. Pfister and Sullivan proved in [10] that $h_{B}(f, B(\mu, f))=$ $h_{\mu}(f)$ for every $\mu \in \mathcal{M}(f)$ (by Proposition 2.1 and Theorem 1.2 in there). Then for the heteroclinic set $H_{f}(\mu, \nu)=B(\mu, f) \cap B\left(\nu, f^{-1}\right)$,

$$
h_{B}\left(f, H_{f}(\mu, \nu)\right) \leq h_{B}(f, B(\mu, f))=h_{\mu}(f) .
$$

Then we cover $M$ by rectangles, say $\left\{P_{1}, \cdots, P_{d}\right\}$ and assume $h_{B}\left(f, B(\mu, f) \cap P_{i}\right)=$ $h_{B}(f, B(\mu, f))$ for some $P_{i}=\left[W_{l o c}^{s}\left(x_{i}\right), W_{l o c}^{u}\left(x_{i}\right)\right]$. Note that $E=B(\mu, f) \cap P_{i}$ is $W_{P_{i}}^{s}$ saturated. By Lemma 5.2 we see $h_{B}\left(f, B(\mu, f) \cap P_{i}\right)=h_{B}\left(f, B(\mu, f) \cap W_{P_{i}}^{u}(y)\right)$ for every $y \in W_{l o c}^{s}\left(x_{i}\right)$.

As observed in Remark 1, we know that $W_{P_{i}}^{u}(y) \subset B\left(\nu, f^{-1}\right)$ for some $y \in W_{\text {loc }}^{s}\left(x_{i}\right)$, since $B\left(\nu, f^{-1}\right)$ is dense and $u$-saturated. So $B(\mu, f) \cap W_{P_{i}}^{u}(y) \subset B(\mu, f) \cap B\left(\nu, f^{-1}\right)=H_{f}(\mu, \nu)$. Then we have 


$$
\begin{aligned}
h_{B}\left(f, H_{f}(\mu, \nu)\right) & \geq h_{B}\left(f, B(\mu, f) \cap W_{P_{i}}^{u}(y)\right) \\
& =h_{B}\left(f, B(\mu, f) \cap P_{i}\right)=h_{B}\left(f, B(\mu, f) \cap P_{i}\right)=h_{\mu}(f) .
\end{aligned}
$$

Combing (5.3) and (5.4), we see $h_{B}\left(f, H_{f}(\mu, \nu)\right)=h_{\mu}(f)$.

Note that $H_{f^{-1}}(\nu, \mu)=H_{f}(\mu, \nu)$ and $h_{\nu}(f)=h_{\nu}\left(f^{-1}\right)$. Therefore

$$
h_{B}\left(f^{-1}, H_{f}(\mu, \nu)\right)=h_{B}\left(f^{-1}, H_{f^{-1}}(\nu, \mu)\right)=h_{\nu}\left(f^{-1}\right)=h_{\nu}(f) .
$$

So if $h_{\mu}(f) \neq h_{\nu}(f)$ for some $\mu, \nu \in \mathcal{M}(f)$, then $h_{B}\left(f, H_{f}(\mu, \nu)\right) \neq h_{B}\left(f^{-1}, H_{f}(\mu, \nu)\right)$. This completes the proof.

\section{REFERENCES}

[1] J. Aaronson, An introduction to infinite ergodic theory, Math. Surveys Monogr. 50, Amer. Math. Soc., Providence, RI, 1997.

[2] A. Avila and J. Bochi, Nonuniform hyperbolicity, global dominated splittings and generic properties of volume-preserving diffeomorphisms, Tran. Amer. Math. Soc. (to appear).

[3] R. Bowen, Topological entropy for noncompact sets, Trans. Amer. Math. Soc. 184 (1973), 125-136.

[4] K. Burns and A. Wilkinson, On the ergodicity of partially hyperbolic systems, Annals of Math. 171 (2010) 451-489.

[5] D. Dolgopyat and A Wilkinson, Stable accessibility is $C^{1}$ dense, Geometric methods in dynamics. II. Astérisque No. 287 (2003), xvii, 33-60.

[6] B. M. Gurevic and V. I. Oseledec, Gibbs distributions, and the dissipativity of C-diffeomorphisms, (Russian) Dokl. Akad. Nauk SSSR 209 (1973), 1021-1023. English translation: Soviet Math. Dokl. 14 (1973), 570-573.

[7] P. Halmos, Invariant measures, Annals of Math. 48 (1947), 735-754.

[8] P. Halmos, Lectures on ergodic theory, The Mathematical Society of Japan, Tokyo, 1956.

[9] V. A. Kaimanovich, Hopf Decomposition and Horospheric Limit Sets, Annales Academiæ Scientiarum Fennicæ, Mathematica, 35 (2010), 335-350.

[10] C. E. Pfister and W. G. Sullivan, On the topological entropy of saturated sets, Ergod. Th. Dynam. Sys. 27 (2007), 929-956.

[11] Y. Sinǎ̌, Markov partitions and C-diffeomorphisms, Func. Anal. Appl. 2 (1968), 61-82.

[12] Z. Xia, Hyperbolic invariant sets with positive measures, Discrete Contin. Dyn. Syst. 15 (2006), no. $3,811-818$.

[13] P. Zhang, Partially hyperbolic sets with positive measure and ACIP for partially hyperbolic systems, Discrete Contin. Dyn. Syst. 32 (2012), 1435-1447.

E-mail address: pfzh311@gmail.com

School of Mathematical Sciences, Peking University, Beijing 100871, China 